\title{
LA DENOMINACIÓN DE ORIGEN CARIÑENA COMO SISTEMA PRODUCTIVO LOCAL: REDES DE EMPRESAS, CULTURA Y GOBERNANZA ${ }^{1 *}$
}

\author{
Climent-López, E., Escalona-Orcao, A.I., Loscertales-Palomar, B. \\ y Molina-Fuster, T. \\ Universidad de Zaragoza
}

\begin{abstract}
Resumen: Cariñena y su entorno es un territorio que puede calificarse como sistema productivo local, pues cuenta con una red de empresas vinculadas entre sí por relaciones de mercado -especialmente la integración de viticultura y vinicultura por medio de las cooperativas- y por relaciones de cooperación, de las que la denominación de origen es el resultado más patente. El análisis de un conjunto de indicadores calculados a partir de estadísticas oficiales y de una serie de entrevistas semiestructuradas a los principales agentes ha permitido aproximarse al conocimiento de determinados elementos cognitivos y de organización del sistema. Así, se han detectado un bajo nivel de cultura técnica, un alto nivel de cultura empresarial y una baja densidad institucional, que se refleja en unas débiles manifestaciones de gobernanza en red.
\end{abstract}

Palabras clave: sistema productivo local, red de empresas, cultura técnica, cultura empresarial, gobernanza

\begin{abstract}
Cariñena and its environs are a territory that can be considered as a local productive system, since there is a network of enterprises linked by trading relations -specially the integration of vine growing and wine production into cooperatives- and by cooperation relationships, being the Guarantee of Origin and Quality of Wine the clearest outcome. The analysis of a set of indicators calculated from official statistics and of a set of semi-structured interviews to the main agents has led to the knowledge of certain cognitive and organisational components of the system. Those identified are a low level of technical culture, a high level of entrepreneurial culture and a low institutional density, reflected in weak outcomes of network governance.
\end{abstract}

\footnotetext{
* Recibido: 5-11-07. Aceptado: 14-12-07.

${ }^{1}$ El texto ofrece resultados del proyecto de investigación "Desarrollo territorial, redes institucionales y procesos de innovación socioeconómica en Aragón" (BSO2003-07603-C08-04/CPSO), financiado por el Ministerio de Educación y Ciencia y el FEDER, dentro del Plan Nacional de Investigación Científica, Desarrollo e Innovación Tecnológica.
} 
Key words: Local productive system, entrepreneurial network, technical culture, entrepreneurial culture, governance.

\section{Introducción}

Un sistema productivo local (SPL), siguiendo las pautas generales de la teoría general de sistemas, es un conjunto de elementos -básicamente empresas, puesto que se trata de un sistema productivo- que interactúan a escala local, de manera que cualquier cambio en alguno de los elementos o en sus posiciones relativas repercute en el conjunto. Para que se produzca interacción no es condición suficiente que las empresas estén localizadas juntas; es menester que se dediquen a las mismas actividades o a actividades conexas.

Atendiendo a ciertas características de las empresas y a las relaciones que mantienen entre sí, podría decirse que hay dos grandes tipos de SPL: los que giran en torno a una gran empresa y los formados por un amplio número de pequeñas y medianas empresas (PYME). Los primeros se rigen por un principio de organización jerárquico, en el que la gran empresa programa su propia producción y además dirige la de las demás empresas, pequeñas y medianas, que son proveedores directos o indirectos suyos. En los segundos, al ser todas las empresas pequeñas y medianas, no hay ninguna que controle a las demás, rigiéndose por un principio de organización mercantil: es el mercado el que en última instancia organiza y dirige la producción de las empresas. Teniendo en cuenta el diferente principio de organización de cada tipo, pero dado que en ambos hay implicada una pluralidad de empresas interrelacionadas, se suele decir que los primeros son "empresas-red" y los segundos "redes de empresas".

El interés que han despertado los SPL de PYME ha sido muy alto, dando lugar a una abundante bibliografía. ${ }^{2}$ Una parte fundamental de dicho interés radica en que la mayoría de ellos son excelentes ejemplos de desarrollo local, por lo que su estudio aporta conocimiento útil para la puesta en práctica de iniciativas de desarrollo basadas en la movilización de los recursos locales.

La localidad aragonesa de Cariñena, junto con su entorno, está estrechamente ligada a la vitivinicultura, actividad en la que presenta una marcada especialización productiva desde tiempo inmemorial. Dicha especialización es indicadora de la exis-

\footnotetext{
${ }^{2}$ Entre la que puede citarse, sin ninguna pretensión de exhaustividad, Garofoli (1992), Courlet et Pecqueur (1992), Courlet (2001) y Lombardi (2003); en relación con España pueden consultarse Climent (1997) y Sánchez (1999)
} 
tencia de una concentración de empresas dedicadas a la misma actividad, lo cual permite plantear la hipótesis de que el territorio de la Denominación de Origen Cariñena es un SPL, por lo que su estudio desde ese enfoque teórico puede ofrecer aportaciones originales al conocimiento de la misma.

\section{El modelo teórico del sistema productivo local de pequeña y mediana empresa}

En los primeros trabajos, tanto teóricos como empíricos, sobre los SPL de PYME, el interés de los investigadores se centró en los aspectos más estrictamente económicos, poniendo el acento en las relaciones entre las empresas. Pero, progresivamente, el foco de atención se fue desplazando hacia los aspectos cognitivos y de organización de los mismos, pasando a un plano central otros agentes territoriales diferentes de las empresas e incorporando aspectos relacionados con el comportamiento de las personas, el funcionamiento de las instituciones e incluso los sistemas de valores.

Las primeras investigaciones ponían el acento en las relaciones de mercado que las empresas mantienen entre sí, tales como la simple competencia, en el caso de las dedicadas a la misma actividad, la relación proveedor-cliente entre las que se dedican a actividades ubicadas en fases consecutivas de la cadena de producción de un determinado bien, la subcontratación de tareas -en sus diversas formas- o la prestación de determinados servicios.

Pero no pasó inadvertido desde el principio el hecho de que la concentración en un espacio local de una red de empresas dedicadas a la misma rama de actividad económica genera unos activos intangibles de carácter cognitivo; a ellos se refirió Marshall -en sus escritos sobre distritos industriales de finales del siglo XIX- con el nombre de atmósfera industrial. En la medida en que el conocimiento sobre los SPL ha ido avanzando se ha ido profundizando en el conocimiento de dichos intangibles:

La atmósfera industrial es básicamente una cultura o saber hacer específico, tanto técnico como empresarial, que se encuentra en cada SPL, pero no fuera de él, y que se adquiere fundamentalmente en las empresas, difundiéndose de unas a otras rápidamente, sin barreras (Albertos, 2002). Dicho saber hacer específico se aprende en la práctica laboral (learning by doing) y se difunde de unas empresas a otras por dos vías principales: el mercado de trabajo y las cadenas de proveedores y clientes que comparten las empresas. A poco evolucionados que estén, y más aún si cuentan con una cierta tradición a sus espaldas, los SPL disponen de trabajadores con una formación técnica específica, bien adaptada a las necesidades del sistema y difícil de encontrar fuera de él; dichos trabajadores muestran una alta propensión a convertirse en 
empresarios. Por otra parte, se ha constatado que en los SPL de PYME los contactos formales e informales entre los empresarios son frecuentes, lo cual abre otra vía, no menos importante, de difusión del saber hacer específico, tanto técnico como empresarial.

En los SPL de PYME, como en todas partes, las empresas se ven enfrentadas continuamente a problemas, para los que van buscando soluciones de forma sistemática, normalmente por el procedimiento de ensayo y error; cada una va dando sus propias respuestas, distintas unas de otras. La ventaja de que disfrutan las empresas insertas en SPL es que la rápida difusión de la información dentro del sistema les permite aprender rápidamente, tanto de su experiencia como de la ajena, desechando las soluciones menos acertadas y seleccionando las más eficaces, a partir de las cuales se plantean nuevos ensayos. De esta manera se desarrolla un proceso continuo de aprendizaje colectivo cuyo resultado es el saber hacer específico.

En los SPL de PYME menos evolucionados el saber hacer específico puede consistir en una mera repetición rutinaria de procedimientos, pero en los que han alcanzado cierta madurez va cambiando a lo largo del tiempo en un proceso continuo de construcción y reconstrucción. En otras palabras, en los SPL maduros se vive la innovación como un proceso continuo: todos los años las empresas lanzan al mercado nuevos modelos o nuevos productos e introducen continuamente cambios en los procesos de producción y gestión, a la vez que buscan nuevos mercados de venta y nuevas fuentes de aprovisionamiento.

No obstante, la bibliografía reciente viene señalando que la velocidad de las innovaciones en el contexto económico global está planteando a los SPL nuevos desafíos que no se pueden resolver mediante un proceso continuo de innovaciones pequeñas (innovación incremental). Para incorporar y asimilar las innovaciones de un cierto calado (innovación radical) hace falta disponer de personal de alta cualificación, para cuya formación ya no es suficiente el learning by doing, siendo necesario el concurso de centros especializados de alto nivel. Por eso, por una parte, a las plantillas de las empresas se van incorporando técnicos procedentes de universidades y centros de formación profesional, junto a los trabajadores formados en la fábrica; y, por otra parte, a los despachos de dirección van accediendo gerentes y empresarios con una amplia formación universitaria y una sólida experiencia de gestión previa, junto a los empresarios hechos a sí mismos a partir de una experiencia laboral como asalariados en el SPL o como asociados desde muy jóvenes a la empresa familiar. ${ }^{3}$

\footnotetext{
${ }^{3}$ Así como hace décadas era normal que los hijos de los empresarios se incorporasen a la empresa nada más alcanzar la edad laboral, actualmente los empresarios prefieren que sus hijos vayan a la universidad, realicen estudios de postgrado y adquieran una sólida experiencia de gestión en otras empresas antes de incorporarse a la suya.
} 
La cultura técnica y la cultura empresarial, aunque intangibles, no escapan a la lógica del mercado, si bien no pueden reducirse a ella. Las propias relaciones entre las empresas también desbordan el marco estrictamente mercantil, pues no todas tienen ese carácter: las empresas de un mismo SPL a menudo mantienen entre sí relaciones de cooperación, entre las que cabe incluir las conversaciones informales y la prestación de favores entre empresarios, la organización conjunta por parte de las empresas de las compras y las ventas de determinados productos, la organización de cursos de formación, la asistencia colectiva a ferias, el mantenimiento de ciertos servicios de interés común, la promoción de denominaciones de origen, la creación de laboratorios o centros de investigación aplicada y la constitución de asociaciones empresariales.

La existencia de dichas relaciones de cooperación es un buen indicador de que un SPL no es sólo una red de empresas. Dicha red está inserta en un marco territorial determinado, en el que se tejen complejas relaciones sociales, que van más allá de la producción, pero que tienen una influencia fundamental en ésta. Por eso las investigaciones recientes sobre los SPL no se circunscriben a las empresas y a los fenómenos estrictamente económicos, sino que amplían su campo de visión a otros agentes cuya función específica no es producir. Entre estos agentes se encuentran los poderes públicos, a los que de forma más clara corresponde la regulación del funcionamiento de la sociedad, pero también instituciones privadas o mixtas (privadas y públicas) que a menudo juegan un papel decisivo en dicha regulación. Por gobernanza se entiende la forma en que se articulan entre sí los distintos agentes, públicos y privados, implicados en la regulación del moderno sistema capitalista:

El enfoque en términos de gobernanza parte del principio de que cada sociedad moderna puede analizarse a partir de la combinación de modos de regulación específicos. En la literatura se identifican cinco tipos ideales de regulación de la economía: el mercado (la competencia), la gran empresa (jerarquía), el Estado (la coacción), la comunidad (solidaridad), la asociación de empleadores (la negociación)" (Benko, 2004: 117).

La gobernanza de los SPL de PYME no corresponde a ninguno de estos tipos ideales, pudiendo encontrarse elementos de varios de ellos: el mercado desempeña un papel fundamental, como ya se ha comentado, pero también el estado juega un rol importante, así como la comunidad y las asociaciones profesionales, resultando una compleja combinación de modos de regulación:

Los poderes públicos locales cobran una singular importancia, tanto por su capacidad de acción específica -piénsese en aspectos tan importantes como la ordenación urbanística y, dentro de ella, la preparación de suelo industrial- como por su capacidad de intermediación ante el Estado central. Dada la proximidad de los ayuntamientos a la comunidad que los elige y su fuerte grado de implicación con ella -espe- 
cialmente si se trata de ámbitos rurales- puede afirmarse que poder local y comunidad tienden a identificarse en estos SPL. Por otra parte, las organizaciones locales de empresas, como articuladoras de los intereses empresariales, tienen una gran capacidad para influir en los poderes locales y, por tanto, para intervenir en la regulación del sistema. Para caracterizar esta complejidad de modos de regulación en los SPL de PYME se utiliza frecuentemente la expresión "gobernanza en red" (Caravaca et al., 2003).

El campo de estudio de los SPL, en conclusión, se ha ensanchado notablemente: de las relaciones interempresariales de mercado a las de cooperación, del aprendizaje en las empresas a la innovación e incorporación de nuevo conocimiento, de las empresas al conjunto de agentes económico-sociales. Pero además se intenta establecer conexiones con valor explicativo entre los distintos elementos considerados: así, se puede suponer que una elevada densidad institucional -una pluralidad de agentes socioeconómicos sólidamente conectados entre sí- estará correlacionada positivamente con la cooperación interempresarial y con la capacidad de aprendizaje y creación de nuevo conocimiento.

\section{Metodología}

En el marco teórico delimitado en el apartado anterior se aborda el estudio de la Denominación de Origen Cariñena como sistema productivo local: en primer lugar se caracteriza su funcionamiento económico, especialmente por lo que respecta a las relaciones entre las empresas, pasando después a analizar los elementos cognitivos y de organización relacionados con el sistema en conjunto: cultura técnica y empresarial por un lado, redes y gobernanza por otro. Como conclusión se intenta establecer correlaciones significativas entre las variables consideradas.

Para caracterizar las relaciones entre las empresas del SPL de Cariñena se intentó en un primer momento realizar una encuesta a todas las bodegas de la Denominación de Origen (DO), pero no pudo obtenerse un número suficiente de cuestionarios debidamente cumplimentados, por lo que se hizo posteriormente otra aproximación metodológica, a partir de entrevistas semiestructuradas, realizadas a gerentes de empresas y representantes de asociaciones e instituciones. La información así obtenida es básicamente cualitativa.

Por lo que respecta a los componentes intangibles de carácter cognitivo, es decir, la cultura técnica y la cultura empresarial, se han estudiado a partir de una serie de indicadores estadísticos, complementados con la información cualitativa obtenida de las entrevistas semiestructuradas: 
Para medir la cultura técnica se han calculado cuatro variables, que posteriormente se han resumido en un indicador de síntesis. Dichas variables son:

1. Porcentaje de la población ocupada incluida en los códigos 2 y 3 de la Clasificación Nacional de Ocupaciones de 1994 (CNO-94) en el censo de 2001 (el código 2 corresponde a profesiones asociadas a titulaciones universitarias y el código 3 a técnicos y profesionales de apoyo).

2. Población con estudios universitarios respecto a la población ocupada (expresado en porcentaje) en el censo de 2001.

3. Población que cursa estudios de formación profesional respecto a la población del grupo de edad de 15 a 19 años (expresado en porcentaje) en el censo de 2001.

4. Población que cursa estudios universitarios respecto a la población del grupo de edad de 20 a 29 años (expresado en porcentaje) en el censo de 2001.

Para mejor interpretar los valores de las variables, se tomaron como referencia los del conjunto de España, procediendo a estandarizar aquéllos mediante la fórmula siguiente:

$$
\mathrm{VS}=\left(\mathrm{V}_{\mathrm{SPL}}-\mathrm{V}_{\mathrm{E}}\right) / \mathrm{V}_{\mathrm{E}}
$$

(VS es el valor estandarizado, $\mathrm{V}_{\mathrm{SPL}}$ es el valor de la variable en el SPL y $\mathrm{V}_{\mathrm{E}}$ el valor de la variable en España).

Las cuatro variables se resumen en un indicador de síntesis, que se ha obtenido mediante el siguiente procedimiento: primero, se ha calculado la media aritmética de los valores estandarizados de las variables 3 y 4 . Segundo, se ha calculado la media aritmética de ésta y los valores estandarizados de las variables 1 y 2, siendo éste el valor del indicador de síntesis.

Para medir la cultura empresarial se han calculado cuatro variables, habiéndose construido un indicador de síntesis con las tres primeras, que son:

1. Empresarios no agrarios con asalariados respecto a la población ocupada no agraria (expresado en porcentaje) en el censo de 2001.

2. Empresarios no agrarios con asalariados y estudios de tercer grado respecto a la población total con estudios de tercer grado (expresado en porcentaje) en el censo de 2001.

3. Empresarios no agrarios con asalariados y educación de tercer grado respecto al número total de empresarios no agrarios (expresado en porcentaje) en el censo de 2001.

Los valores de dichas variables se han estandarizado siguiendo el mismo procedimiento que en las relacionadas con la cultura técnica. El indicador de síntesis de cul- 
tura empresarial se ha obtenido calculando la media aritmética de los valores estandarizados de las tres variables.

La cuarta variable para medir la cultura empresarial es la tasa de natalidad de empresas industriales, que se ha calculado relacionando el número de empresas nuevas creadas en el último año con el número de empresas existentes en dicho año. El primer dato se obtuvo del Registro de Establecimientos Industriales, si bien para evitar la influencia de alguna posible situación coyuntural se tomó como valor del último año la media aritmética de los correspondientes a 2003 y 2004. El segundo se obtuvo de la base de datos de empresas de las Cámaras de Comercio (CAMERDATA), considerando sólo las industriales y excluyendo los trabajadores autónomos y las empresas sin asalariados. La tasa de natalidad es la proporción de nuevas empresas (primer dato) sobre el total de empresas (segundo dato) expresado en porcentaje. El valor así obtenido se ha estandarizado por el procedimiento ya indicado, pero tomando como referencia la tasa de Aragón, dada la imposibilidad de obtener para el conjunto del país el número de nuevas empresas creadas.

Pasando de los aspectos cognitivos a los organizativos, para el estudio de éstos se ha recurrido también a las entrevistas semiestructuradas, por medio de las cuales se ha obtenido información sobre los agentes actuantes en el SPL de Cariñena, sobre el carácter e intensidad de sus relaciones mutuas y sobre la gobernanza.

Se ha considerado que un agente actúa en el territorio cuando interviene de manera directa y con una influencia efectiva en las actividades económicas del SPL. La existencia de relaciones entre dos agentes se ha constatado cuando ha habido coincidencia en la respuesta afirmativa de ambos y la intensidad y frecuencia de sus relaciones se ha determinado como el valor medio de las respuestas de uno y otro.

Se ha medido la densidad institucional del SPL por medio del índice de conectividad relativa, que valora el porcentaje de relaciones efectivamente existentes respecto al número de relaciones posibles, en la situación ideal en que estuvieran presentes en el SPL todas las categorías relevantes de agentes y que éstos mantuvieran relaciones todos con todos. Los tipos de agentes que se han considerado relevantes, siguiendo la bibliografía citada en el marco teórico (especialmente Caravaca et al., 2003), son nueve: administración local, administración regional, empresas, organizaciones empresariales, sindicatos, agentes sociales y comunitarios, centros de investigación, centros de formación especializada y centros de servicios avanzados a las empresas. La fórmula matemática para el cálculo del índice de conectividad relativa es la siguiente:

$$
\mathrm{I}_{\mathrm{CR}}=100 * 2 \mathrm{R} /[\mathrm{A}(\mathrm{A}-1)]
$$


$\left(\mathrm{I}_{\mathrm{CR}}\right.$ es el índice de conectividad relativa; $\mathrm{R}$ es el número de relaciones constatadas entre las categorías de agentes actuantes en el territorio; A es el número de categorías relevantes de agentes, es decir, 9).

Una vez conocidos los agentes actuantes en el SPL y la densidad institucional de éste, la investigación (siempre mediante entrevistas semiestructuradas) ha ido dirigida a indagar sobre la gobernanza de la zona. La manera en que una red local de agentes puede influir en la regulación de la actividad económica local es forzosamente limitada: no se puede olvidar que la economía local se ubica en el contexto de una economía de mercado abierta y global y en el marco de unas instituciones estatales y supraestatales -la Unión Europea- con amplios poderes de regulación. De lo que se trata, por tanto, es de indagar qué actuaciones se han puesto en marcha en el ámbito local para que la economía local se integre con éxito en el contexto de los mercados globales, asumiendo el marco regulador español y europeo. Para ello se ha valorado si los agentes locales han realizado un diagnóstico económico del territorio, si han creado instituciones de desarrollo local y si han puesto en marcha actividades económicas conjuntas.

\section{La denominación de origen Cariñena y su red de empresas vitivinícolas}

La DO Cariñena está integrada por 14 municipios, que albergan a cerca de 12.000 habitantes. Cariñena, con algo más de 3.000, es el más poblado de todos y en él se concentra el 28 por ciento de la población total, no habiendo ningún otro municipio que supere el umbral de los 2.000 (tabla 1). Puede afirmarse, por tanto, que se trata de un espacio de carácter rural.

Se trata de la DO vitivinícola más extensa de Aragón, con más de 14.000 hectáreas dedicadas al cultivo de la vid, y también la más antigua, con 75 años de existencia a sus espaldas. En ella se hallan registradas 52 bodegas. En ambas magnitudes destaca el municipio de Cariñena, que concentra el 24 por ciento de la superficie de viñedo y el 31 por ciento de las bodegas de la DO (tabla 1 y figura 1).

La especialización productiva de la zona queda de manifiesto tomando en consideración los siguientes datos: por una parte, la superficie ocupada por el viñedo equivale al 30 por ciento de las tierras cultivadas, pero ese valor medio se eleva por encima del 50 por ciento en Cariñena y en la mayor parte de sus municipios limítrofes, en lo que podría considerarse el corazón de la DO; de ahí se desprende que la viticultura tiene un peso muy fuerte en la economía agraria local. Por otra parte, las 
Tabla 1. Población, superficie cultivada y bodegas de la Denominación de Origen Cariñena.

\begin{tabular}{lrrrrr}
\hline MUNICIPIO & $\begin{array}{c}\text { Habitantes } \\
(2006)^{*}\end{array}$ & $\begin{array}{c}\text { Has. cultivo } \\
\text { vid } \\
(1999)^{* *}\end{array}$ & $\begin{array}{c}\text { Has. } \\
\text { labradas } \\
(1999)^{* *}\end{array}$ & $\begin{array}{c}\text { Vid/ } \\
\text { labradas } \\
(1999)(\%)^{* *}\end{array}$ & $\begin{array}{c}\text { Bodegas } \\
\text { DO**** }\end{array}$ \\
\hline Aguarón & 798 & 1.475 & 1.937 & 76,15 & 3 \\
Aladrén & 62 & 36 & 167 & 21,56 & 0 \\
Alfamén & 1.588 & 1.399 & 8.291 & 16,87 & 4 \\
Almonacid de la Sierra & 853 & 1.281 & 3.268 & 39,20 & 13 \\
Alpartir & 576 & 111 & 1.023 & 10,85 & 0 \\
Cariñena & 3.277 & 3.359 & 6.588 & 50,99 & 16 \\
Cosuenda & 377 & 617 & 1.059 & 58,26 & 1 \\
Encinacorba & 279 & 425 & 1.063 & 39,98 & 1 \\
Longares & 879 & 2.329 & 4.917 & 47,37 & 3 \\
Mezalocha & 260 & 186 & 4.920 & 3,78 & 2 \\
Muel & 1.200 & 210 & 5.845 & 3,59 & 4 \\
Paniza & 742 & 1.751 & 2.451 & 71,44 & 1 \\
Tosos & 215 & 487 & 3.102 & 15,70 & 3 \\
Villanueva de Huerva & 590 & 599 & 3.591 & 16,68 & 1 \\
TOTAL & 11.696 & 14.265 & 48.222 & 29,58 & 52 \\
\hline
\end{tabular}

"Fuente: http://bonansa.aragon.es:81/estadis/base_ter/bas_inicio.jsp (Instituto Aragonés de Estadística: Padrón Municipal de Habitantes).

"** Fuente: http://bonansa.aragon.es:81/estadis/base_ter/bas_inicio.jsp (Instituto Aragonés de Estadística: Censo Agrario).

**** Fuente: www.docarinena.es (Denominación de Origen Cariñena).

estadísticas de afiliación a la seguridad social muestran que, en el conjunto de la DO, la industria de productos alimenticios y bebidas da empleo al 44 por ciento de los afiliados del sector industrial y al 23 por ciento del total. ${ }^{4}$

Por otra parte, el peso del sector vitivinícola local en el conjunto regional queda de relieve si se tiene en cuenta que el 36 por ciento de la superficie dedicada en Aragón al cultivo de la vid y el 25 por ciento de las empresas dedicadas a la elaboración de vino en la región se localizan en la zona. ${ }^{5}$

\footnotetext{
${ }^{4}$ La fuente es el Instituto Aragonés de Estadística (http://bonansa.aragon.es:81/estadis/base_ter/bas_inicio.jsp) y los datos corresponden al año 2002; aunque no aparece desagregada en la fuente original, la industria vinícola constituye el grueso de la rama de productos alimenticios y bebidas de la zona.

${ }^{5}$ Las cifras de superficie de viñedo proceden del censo agrario de 1999. Respecto a las de bodegas que elaboran vino, para que la comparación resulte válida, tanto las cifras de la DO como las totales de Aragón se han tomado de la misma fuente, la base de datos de empresas de las Cámaras de Comercio e Industria (www.camerdata.es); no obstante, es preciso hacer notar que es mayor el número de bodegas locales registradas en la DO que en CAMERDATA (52 frente a 40).
} 


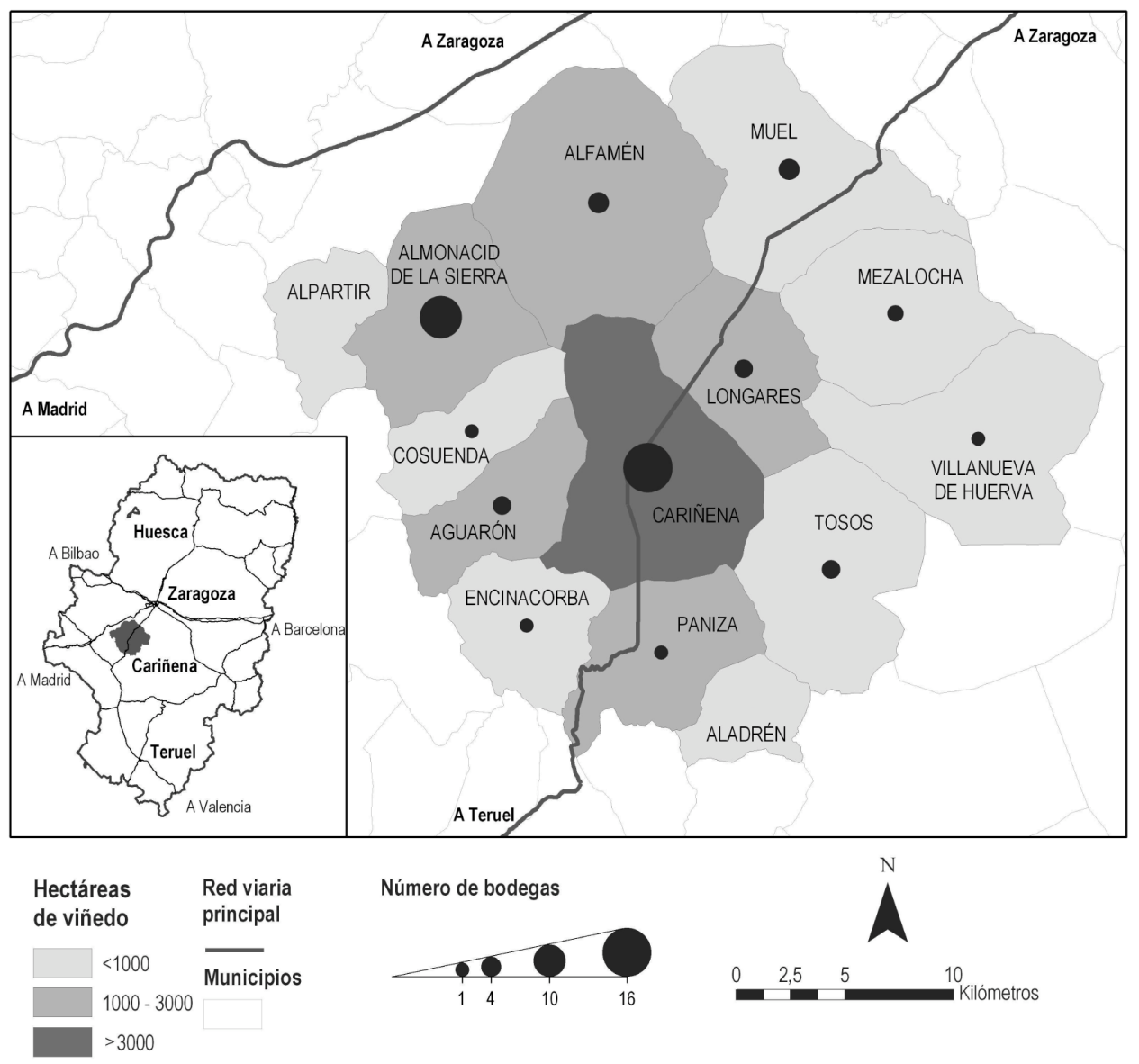

Figura 1. Viñedos y bodegas en la Denominación de Origen Cariñena.

De lo anterior se desprende que la DO Cariñena reúne los requisitos de especialización productiva y peso económico suficientes para considerarla un SPL. A ello se añade el hecho de que todas sus empresas vinícolas encajan sin ninguna duda en la categoría de PYME, pues ninguna de ellas supera los 100 empleados y sólo dos se encuentran entre 50 y 100, quedando las demás por debajo de 50.

Las entrevistas semiestructuradas a que se ha hecho referencia en el apartado 3, dedicado a la metodología, han puesto de manifiesto que la mayoría de las empresas de elaboración de vino son endógenas. Las más importantes, atendiendo al volumen de producción, son las 10 cooperativas creadas por los propios viticultores de la zona. 
Las empresas productoras de vino, de acuerdo con la normativa de la DO, se abastecen de la materia prima -las uvas- dentro del territorio del propio SPL, en lo que constituye un claro proceso de integración local de las diferentes fases de elaboración de un bien de consumo. Las bodegas cooperativas son el principal instrumento de dicha integración, puesto que sus socios son a la vez los viticultores que aportan la materia prima; pero las bodegas particulares también adquieren sus uvas en el entorno local.

La muestra más patente de la existencia de sólidas relaciones de cooperación entre las empresas es la existencia de una DO, en tanto que implica la aprobación y cumplimiento de unas determinadas normas por parte de todas ellas. La DO se rige por medio de un Consejo Regulador, que está constituido de la siguiente manera: seis vocales en representación del sector vitícola, es decir, de los titulares de viñedos inscritos en el Registro de Viñas de la DO; seis vocales en representación del sector vinícola, es decir, de los titulares de bodegas inscritos en los registros de la DO; dos vocales designados por el Departamento de Agricultura del Gobierno de Aragón entre personas con especiales conocimientos en la materia; finalmente, como máximo representante de la institución está el Presidente, designado por el Consejero de Agricultura del Gobierno de Aragón a propuesta del propio Consejo Regulador.

El entramado empresarial se completa con algunas empresas dedicadas a tareas complementarias y auxiliares del cultivo de la vid y la elaboración del vino, como, por ejemplo, la producción de soportes para el cultivo de la vid en espaldera -que es el procedimiento generalizado actualmente- o la fabricación de maquinaria industrial para el sector vitivinícola. No obstante, las empresas industriales de este tipo presentes en la zona se pueden contar con los dedos de una mano.

En definitiva, la investigación cualitativa ha puesto de relieve la existencia de relaciones de mercado y de cooperación entre las empresas y ha permitido caracterizarlas. A la vista de todo ello, puede afirmarse que la DO Cariñena constituye un SPL de PYME que integra una red de empresas agrícolas y agroalimentarias estrechamente conectadas entre sí y que además mantienen unas sólidas relaciones de cooperación, formalizadas por medio de un Consejo Regulador.

\section{Cultura técnica y cultura empresarial}

En la primera parte de la tabla 2 se recogen los valores de las variables utilizadas para medir la cultura técnica del territorio, según se ha indicado en el apartado 3, dedicado a la metodología. Puede comprobarse que en todos los casos los valores de 
Cariñena son inferiores a los valores medios del conjunto de España, lo cual da lugar a valores estandarizados negativos:

El del porcentaje de profesionales y técnicos de apoyo en la población ocupada queda ligeramente por debajo de $-0,5$ lo que significa que dicho porcentaje en Cariñena es inferior a la mitad de la media nacional: 11,3 frente a 23 por ciento, respectivamente. Los valores estandarizados de las demás variables no son tan bajos, pero se ve claro que el porcentaje de personas con estudios universitarios y el de jóvenes que estudian en la universidad o en los centros de formación profesional se encuentran por debajo de la media nacional. Como consecuencia de ello el indicador de síntesis arroja un valor negativo $(-0,32)$. Puede afirmarse, por ello, que se trata de un SPL que tiene un nivel de cultura técnica bajo con relación al conjunto de España.

En la segunda parte de la tabla 2 pueden verse los valores estandarizados de las variables que miden la cultura empresarial. El porcentaje de empresarios con asalariados dentro de la población ocupada no agraria del SPL es casi idéntico -en realidad, muy ligeramente inferior- a la media nacional. Pero el porcentaje de empresarios no agrarios con asalariados dentro de la población con estudios universitarios es claramente superior a la media nacional. Del mismo modo, dentro de los empresarios no agrarios el porcentaje de los que tienen estudios universitarios es superior a la media nacional. Los valores estandarizados de estas variables, en consecuencia, son positivos, excepto el de la primera que es negativo sólo por unas pocas centésimas. El indicador de síntesis, lógicamente, es positivo, lo cual significa que el nivel de cultura empresarial de Cariñena está por encima de la media nacional. En otras palabras, en Cariñena hay una propensión alta a considerar la actividad empresarial como ocupación y medio de vida, sobre todo para la población con estudios universitarios.

La variable complementaria de cultura empresarial, la tasa de natalidad, presenta un valor estandarizado positivo muy alto, lo que significa que en el SPL se han creado más empresas que en el conjunto regional, en términos proporcionales al número de empresas existentes (tabla 2). Este dato refuerza la imagen de Cariñena como territorio con una elevada cultura empresarial.

En conclusión, empezando por el aspecto más positivo, Cariñena posee, como ocurre en la generalidad de los SPL, una cultura empresarial elevada, lo que equivale a decir que existe una propensión relativamente alta a crear empresas o, en otras palabras, que la actividad empresarial es altamente valorada como ocupación y modo de vida por la población. En este caso, además, es la población con estudios superiores la que realmente muestra dicha propensión, lo cual implica la existencia de una proporción relativamente elevada de empresarios con estudios universitarios. Éste es, sin duda, uno de los mejores activos intangibles con que cuenta la DO Cariñena. 
Tabla 2. Cultura técnica y empresarial del sistema productivo local de Cariñena.

\begin{tabular}{llrrr}
\hline & VARIABLES E INDICADORES & $\begin{array}{c}\text { Cariñena } \\
(\%)\end{array}$ & $\begin{array}{c}\text { España } \\
(\%)\end{array}$ & $\begin{array}{r}\text { Valor } \\
\text { estándar }\end{array}$ \\
\hline Cultura & Profesionales y técnicos de apoyo & 11,32 & 23,01 & $-0,51$ \\
técnica & Población con estudios universitarios & 16,59 & 20,64 & $-0,20$ \\
& Población que cursa formación profesional & 15,02 & 18,47 & $-0,19$ \\
& Población que cursa estudios universitarios & 17,32 & 24,69 & $-0,30$ \\
& Indicador de síntesis de cultura técnica & - & - & $-0,32$ \\
\hline Cultura & Empresarios no agrarios con asalariados & 5,12 & 5,59 & $-0,08$ \\
empresarial & Empresarios con estudios de tercer grado & 16,57 & 12,14 & 0,37 \\
& Población con estudios universitarios & & & \\
& y empresarios & 5,51 & 3,08 & 0,79 \\
& Indicador de síntesis de cultura empresarial & - & - & 0,36 \\
& Tasa de natalidad de empresas industriales & 21,43 & $6,56\left(^{*}\right)$ & $2,27\left(^{*}\right)$ \\
\hline
\end{tabular}

" Tasa de Aragón; no ha sido posible calcular la tasa de España. El valor estándar, por tanto, está calculado respecto a la tasa de Aragón.

El contrapunto es el bajo nivel de cultura técnica, que puede resultar una seria traba para la evolución del SPL. Como se ha indicado en el apartado segundo, la globalización acelera los procesos de innovación, lo cual implica que para mantenerse al día no basta con el conocimiento práctico tradicional difuso en el SPL, siendo necesario contar con un elevado nivel de cultura técnica para incorporar el nuevo conocimiento que se crea en el mundo y saber adaptarlo a las características de la producción local. La constante evolución de los gustos respecto al vino y la incorporación creciente de nuevos países productores a los mercados internacionales constituyen serios desafíos para las DO tradicionales, como la de Cariñena. Un elevado nivel de cultura técnica, traducido en una mayor presencia de profesionales de alto nivel (agrónomos, químicos, enólogos, expertos en marketing y otros), sería uno de los mejores activos para hacer frente a dicho desafío.

\section{Redes y gobernanza}

La figura 2 representa la red de agentes del SPL de Cariñena, conocida por medio de entrevistas semiestructuradas, según se ha explicado en el apartado tercero. Cada círculo de la figura corresponde a una categoría de agente cuya presencia en los SPL -en general, no en éste en concreto- ha sido constatada fehacientemente por la bibliografía disponible, lo cual no quiere decir que todos ellos estén presentes en todos los SPL. El gráfico de Cariñena precisamente indica cuáles de ellos están pre- 
sentes (los representados con un círculo de color gris) y cuáles no (los representados con un círculo vacío). Los círculos grises están unidos entre sí por medio de líneas, que representan las relaciones existentes entre los tipos de agentes; dichas líneas han sido dibujadas con distintos grosores, en función de la intensidad y frecuencia de las relaciones: cuanto más intensa y frecuente es la relación mayor es el grosor de la línea.

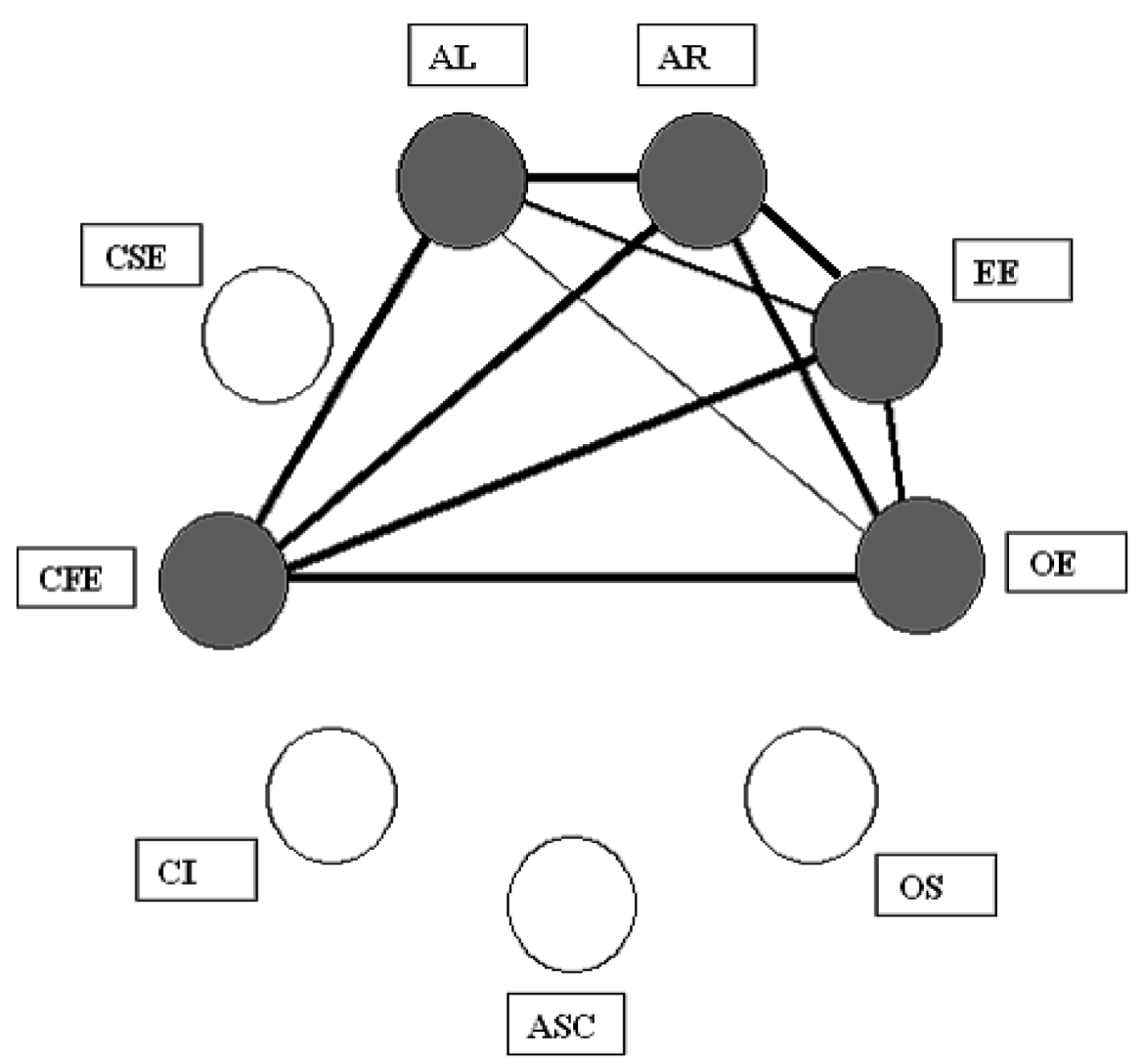

Figura 2. Red de agentes del sistema productivo local de Cariñena.

AL: Administración local

EE: Empresas

OS: Organizaciones sindicales

CI: Centros de investigación

CSE: Centros de servicios avanzados a las empresas
AR: Administración regional

OE: Organizaciones empresariales

ASC: Agentes sociales y comunitarios

CFE: Centros de formación especializada 
Los agentes presentes en la red responden a cinco de los nueve tipos posibles: administración local y regional, empresas, organizaciones empresariales y centros de formación especializada.

La administración local está integrada por los Ayuntamientos de los municipios y por el Consejo Comarcal. Aragón es una de las pocas comunidades autónomas que ha creado una nueva administración de carácter local entre el municipio y la provincia, que es la comarca. No existe coincidencia plena entre la comarca del Campo de Cariñena y el SPL de Cariñena (que, como ya se ha indicado, coincide con el ámbito territorial de la denominación de origen del mismo nombre), pero la mayor parte de los municipios del SPL -y entre ellos los de mayor población y peso económico- están en dicha comarca. Como se trata de una entidad encuadrada dentro de la administración local, se ha incluido también en este grupo de agentes la Agencia de Desarrollo Local gestora del programa PRODER de la Unión Europea.

La administración regional, es decir, el Gobierno de Aragón, se considera agente local en tanto que interviene directamente en la actividad de la zona. En Cariñena lo hace por medio de tres organismos: la Consejería de Agricultura, fuertemente implicada en el Consejo Regulador de la Denominación de Origen (CRDO), según se ha descrito en el apartado cuarto; la Consejería de Educación, que es la responsable del centro de formación especializada, y el Instituto Aragonés de Fomento -la agencia regional de desarrollo- que participa como accionista en una empresa estratégica del SPL de Cariñena. ${ }^{6}$

Ya se ha hecho antes mención a las empresas, por lo que procede referirse a las organizaciones empresariales. En el SPL de Cariñena las más relevantes son: la Unión de Agricultores y Ganaderos de Aragón (UAGA) -que es el sindicato agrario más representativo de los viticultores de la zona y, en consecuencia, el que tiene mayor peso en las cooperativas vinícolas- y el CRDO, en el que, como se ha indicado en el apartado cuarto, están representados tanto los viticultores como las bodegas.

El centro de formación especializada de Cariñena es el Instituto de Educación Secundaria (IES) "Joaquín Costa", dependiente de la administración regional, que dispone de ciclos formativos de Formación Profesional Específica, en concreto de uno de grado medio ("Elaboración de vinos y bebidas") y otro de grado superior ("Industrias alimentarias", con capacitación en vitivinicultura). Se trata de un centro fuertemente relacionado con la actividad económica dominante en la zona, que goza de amplio prestigio, como indica el hecho de que a él acudan alumnos de todo Aragón y de otras comunidades autónomas.

${ }^{6}$ Se trata de "Grandes Vinos y Viñedos", que es una empresa creada en 1997 por cinco de las cooperativas de la DO con el apoyo del Gobierno regional y las principales cajas de ahorro aragonesas; su objeto es canalizar las ventas de dichas cooperativas, el 40 por ciento de las cuales se dirige ya a la exportación. 
Las relaciones entre las categorías de agentes presentes en la red son numerosas, pues los de todas ellas se relacionan con los de las cuatro restantes. Pero, dado que su número es claramente inferior al máximo posible, la densidad institucional del SPL es débil: su índice de conectividad relativa, calculado según lo indicado en el apartado tercero, alcanza el valor 28 , lo que significa que las relaciones realmente existentes entre los agentes presentes en el SPL son el 28 por ciento de las posibles en el modelo ideal.

La red representada en el gráfico de la figura 2 es mixta, pues hay agentes públicos y privados. En el cómputo general de relaciones tienen mayor peso los primeros (60 por ciento de las relaciones) que los segundos (40 por ciento)

La intensidad de las relaciones es alta, como indica el grosor de los trazos en el gráfico, aunque hay un tipo de agente que mantiene nexos más débiles con los demás: la administración local. Respecto a la frecuencia de las relaciones entre los tipos de agentes, puede afirmarse que el 80 por ciento de ellas son frecuentes y estables, siendo el 20 por ciento restante de carácter eventual.

Una vez conocidos los agentes actuantes en el SPL, medida su densidad institucional y construido el grafo que representa la red de relaciones entre aquéllos, se ha indagado, por medio de entrevistas semiestructuradas sobre la gobernanza de la zona, valorando si los agentes locales han realizado un diagnóstico económico del territorio, si han creado instituciones de desarrollo local y si han puesto en marcha actividades económicas conjuntas, tal como se ha indicado en el apartado tercero.

El único estudio elaborado para diagnosticar la situación económica del territorio es el que se hizo como preparación del programa PRODER. Pero ese estudio parece ser conocido o realmente valorado sólo por la Agencia de Desarrollo Local gestora del mismo. En las entrevistas los representantes de las administraciones locales y de las organizaciones empresariales, sorprendentemente, no lo mencionaron, a pesar de que sus instituciones tienen representación en la Junta Directiva de la agencia; tampoco lo mencionó el representante del IES.

Los representantes de las organizaciones empresariales mencionaron en este apartado algunos estudios concretos de carácter sectorial, es decir, específicamente dedicados al sector del vino, pero en realidad no constituyen un diagnóstico del territorio, por lo que no corresponden al tipo de estudio por el que se indagaba.

La creación de la mencionada Agencia de Desarrollo Local ha estado vinculada a los programas de desarrollo rural de la Unión Europea. No ha surgido, por tanto, como iniciativa autónoma para responder a necesidades específicas definidas desde dentro, sino como respuesta a estímulos externos: en la zona de Cariñena se creó inicialmente la "Asociación para el Desarrollo Integral de Campo de Cariñena" 
(ADICCA), que junto con una asociación del mismo tipo de la vecina comarca de Valdejalón integraron después la "Federación de Asociaciones para el Desarrollo Integral de Valdejalón y Campo de Cariñena" (FEDIVALCA), que es la Agencia de Desarrollo Local gestora del programa PRODER. Esta asociación es citada por todos los agentes entrevistados en el SPL, que la conocen y la asocian con el desarrollo local, si bien los representantes del sector vitivinícola afirman que apenas tiene algo que ver con su sector.

Respecto a iniciativas económicas conjuntas, las respuestas dadas por los agentes entrevistados en Cariñena permiten concluir que el sector vitivinícola funciona muy bien vertebrado en torno a sí mismo, con el CRDO como organismo de control e impulso y la UAGA como sindicato agrario representativo de la mayor parte de los viticultores, que a su vez son socios de las cooperativas, claramente dominantes en la zona. Estos agentes, en las entrevistas, no mencionan exactamente iniciativas económicas conjuntas, sino que hacen referencia más bien a un proceso continuo de innovación, tanto en las técnicas de cultivo como en las de elaboración del vino y la comercialización. Cabe citar, no obstante, la creación de un Museo del Vino, ligado al CRDO, iniciativa que, siendo coherente con los intereses estrictos del sector vitivinícola, va más allá de ellos, pues su objetivo central es atraer turistas a la zona, lo que tendrá repercusión en el conjunto del territorio. En esta misma línea va el acuerdo firmado por el CRDO con la Facultad de Filosofía y Letras de la Universidad de Zaragoza para la realización de trabajos de investigación sobre la cultura del vino en todas sus facetas.

Las administraciones locales mencionan como iniciativa económica importante la creación de polígonos industriales, pero la valoración que hacen de ello los demás agentes no es totalmente positiva: los ayuntamientos de Muel, Cariñena y Alfamén han promovido polígonos de este tipo, cuya finalidad principal es atraer empresas de fuera, aprovechando la proximidad del SPL al área metropolitana de Zaragoza y las excelentes comunicaciones por autovía con ella (figura 1). ${ }^{7}$ Los representantes del sector vinícola expresan una cierta sensación de agravio comparativo al respecto, pues lo valoran como un apoyo a empresas de nueva instalación, que vienen de otros lugares (de manera que, igual que han venido, se pueden marchar), mientras que las bodegas, de fuerte arraigo en la comarca (de donde nunca se van a ir), no reciben ni han recibido ningún tipo de ayuda. Esto es una muestra de la falta de sintonía entre algunas instituciones locales y los agentes económicos del sector vitivinícola, que tiene su reflejo en la debilidad de las relaciones entre ambos observada en el gráfico de la figura 2.

\footnotetext{
${ }^{7}$ De hecho, ya se han instalado en la zona algunas empresas foráneas dedicadas a sectores industriales diferentes del vinícola, entre las que destaca una multinacional del sector del mueble establecida en Cariñena, que da empleo a cerca de 500 trabajadores.
} 
Para concluir, por una parte, el SPL de Cariñena adolece de una baja densidad institucional, pues no se ha constatado la presencia activa de algunos tipos de agentes que en otros SPL han desempeñado un papel importante (sindicatos obreros, centros de investigación y de servicios especializados u otro tipo de agentes socio-comunitarios). Esta baja densidad es lógica en espacios rurales de escasa población, como es el caso, y se corresponde con la escasez de manifestaciones de gobernanza en red a que se ha hecho referencia, especialmente, el hecho de que la Agencia de Desarrollo Local existente no haya sido el fruto de una iniciativa endógena, sino la respuesta un tanto forzada a un estímulo externo, la necesidad de captar fondos del programa PRODER. Es sintomático a este respecto que el plan estratégico elaborado para poner en marcha dicha iniciativa comunitaria haya tenido escasa repercusión práctica, puesto que no es mencionado por casi ningún agente local.

Por otra parte, hay que destacar como elemento positivo del SPL la fuerte vertebración interna del sector económico en que está especializado, así como la implicación en él de la administración regional, como reguladora del mismo a través del CRDO, como proveedora de servicios educativos especializados a través del IES y como accionista de una empresa de referencia.

Finalmente, se ha detectado un cierto desencuentro entre los agentes directamente implicados en el sector vitivinícola y la administración local, pues varios ayuntamientos -entre los que se encuentra el de la propia Cariñena- han orientado sus esfuerzos hacia la atracción de empresas foráneas, aprovechando la renta de situación de que dispone la zona. Esta orientación provoca recelos en el sector vitivinícola y puede dar lugar a algún tipo de conflicto.

\section{Conclusiones}

El análisis realizado ha permitido poner de relieve la existencia de una sólida red de PYME, con una fuerte integración vertical de la viticultura, la vinicultura y, en mucha menor medida, otras actividades complementarias, por medio de algunas empresas particulares y, especialmente, de las cooperativas de agricultores, que son las elaboradoras de vino más potentes de la zona.

Cabe relacionar la existencia de dicha red con la elevada cultura empresarial que caracteriza al área y con la intensidad de la cooperación entre las empresas, que se revela de forma palmaria en el hecho de haber alcanzado un alto grado de formalización mediante una de las más veteranas denominaciones de origen de España. 
Dicha red de empresas se integra en un entorno social de baja densidad institucional, en el que a las organizaciones empresariales se une la administración regional, con una participación decisiva en la regulación del sector vitivinícola, a través del CRDO. La administración local, por el contrario, mantiene unas relaciones poco intensas con el sector. No se han identificado otros tipos de agentes económicos y sociales con influencia en la actividad económica, excepto el IES, dependiente de la administración regional.

Esa baja densidad institucional tiene su reflejo en unas débiles manifestaciones de gobernanza en red: una agencia de desarrollo local nacida por estímulos externos, no por una iniciativa endógena, y un plan estratégico de escasa repercusión práctica, con el que el sector vitivinícola no se siente comprometido.

Se ha constatado, finalmente, un bajo nivel de cultura técnica. Ello no impide que en el SPL se desarrolle un proceso continuo de innovación, tanto en el cultivo de la vid como en la elaboración del vino: los sistemas de cultivo han evolucionado -ya se ha hecho referencia a la difusión del cultivo en espaldera- y los vinos que salen al mercado actualmente no son iguales que los de hace décadas.

No obstante, ese bajo nivel podría comprometer la evolución favorable del SPL en el marco de la globalización, que se manifiesta en la incorporación creciente de nuevos productores y en el rápido cambio de los gustos y las modas. A este respecto cabe reseñar dos datos que apelan al optimismo: por una parte, hay una alta proporción de empresarios con estudios superiores y de universitarios con vocación empresarial; por otra parte, la zona cuenta con un centro de formación especializado en el sector del vino (el IES), que goza de un buen prestigio dentro y fuera del SPL, y que mantiene relaciones estrechas con los demás agentes económicos y sociales.

El camino para resolver las carencias señaladas se deduce del propio análisis realizado: en primer lugar, habría que elevar el nivel de cultura técnica mejorando la proporción de jóvenes que cursen estudios universitarios y de formación profesional. En segundo lugar, sería deseable que la administración local se comprometiese de una manera más intensa con el sector vitivinícola, sin que ello implique renunciar a otras políticas orientadas a la diversificación económica y al aprovechamiento de las crecientes rentas de situación que ofrecen la nueva autovía y las nuevas expectativas de desarrollo de la cercana ciudad de Zaragoza. 


\section{Bibliografía}

Albertos Puebla, J.M. (2002). Cultura, innovación y desarrollo local. Boletín de la Asociación de Geógrafos Españoles, 34, 229-244.

Benko, G. (2004). Distritos industriales y gobernanza de las economías locales. El caso de Francia. Economia Industrial, 359, 113-125.

Caravaca, I., González, G. y Silva, R. (2003). Redes e innovación socio-institucional en sistemas productivos locales. Boletín de la Asociación de Geógrafos Españoles, 36, 103-115.

Climent López, E. (1997). Sistemas productivos locales y distritos industriales: el caso de España. Boletín de la Asociación de Geógrafos Españoles, 24, 91-106.

Courlet, C. (2001). Les systèmes productifs localisés. Cahiers d'économie et sociologie rurales, 58-59, 82-103.

Courlet, C. y Pecqueur, B. (1992). Les systèmes industriels localisés en France: un nouveau modèle de développement. En Benko, G. y Lipietz, A. (eds) Les régions qui gagnent. Districts et réseaux: les nouveaux paradigmes de la géographie économique, Paris, Presses Universitaires de France, p. 81-102.

Garofoli, G. (1992). Les systèmes de petites entreprises: un cas paradigmatique de développement endogène. En Benko, G. y Lipietz, A. (eds): Les régions qui gagnent. Districts et réseaux: les nouveaux paradigmes de la géographie économique, Paris, Presses Universitaires de France, p. 57-80.

Lombardi, M. (2003). The evolution of local production systems. Research policy, 32, 1443-1462.

Sánchez Hernández, J.L. (1999). Sistemas productivos locales en la península Ibérica: cinco temas de debate. Anales de Geografia de la Universidad Complutense, 19, 215-235. 